\title{
F100-PW-229 Engine Fault Detection Based on Real Time Data
}

\author{
Vasileios Kolios $^{1}$, Ioannis Templalexis ${ }^{2}$, Ioannis Lionis $^{3}$, Emmanouil Antonogiannakis $^{4}$ and \\ Petros Kotsiopoulos ${ }^{2}$ \\ 1335 Fighting Squadron/116 Combat Wing, Araxos Air Base, Achaia, Greece \\ ${ }^{2}$ Hellenic Air Force Academy, Dekelia Air Base, Attika, Greece \\ ${ }^{3}$ Directory of Applied Research, HAF Electronics Depot, Glyfada Attica, Greece \\ ${ }^{4}$ Support Command, Elefsina Air Base, Attika, Greece
}

\begin{abstract}
Gas turbine engines exhibit very high maintenance costs. Moreover, in the case of aero applications an in-flight engine incidence, shall, by all means, be avoided, a condition that drives total maintenance costs even higher. A measure in favor of balancing these costs is to monitor continuously the variation of engine performance data recorded during flight, establish methods to deduce useful information regarding the engine "health" status and, as a result, take appropriate actions to maintain a good engine operating condition. The current work presents such a method tailored on the "F100-PW-229" engine that is operated by the Hellenic Air Force as the propulsion system of the "F-16 block 52M" aircraft [3]. CEDATS and MS Excel were the computational tools used for the current engine performance study. CEDATS is a software developed for the engine users. It provides basic data trend monitoring functions and engine fault warnings. It is well known that there is always space for improvement for such health monitoring tools since there are cases where engine operating faults are not captured. Within the frame of the current work, a data post - processing method on the engine performance data time series was applied using MS Excel, in order to raise early warnings of an uncaptured compressor operating fault.
\end{abstract}

\section{F100-PW-229 Engine Maintenance}

\subsection{Maintenance Philosophy}

On Condition Maintenance (OCM) is a maintenance strategy that monitors the actual condition of an asset to decide what maintenance needs to be done. OCM dictates that maintenance should only be performed when certain indicators show signs of decreasing performance or upcoming failure. Checking a machine for these indicators may include 
non-invasive measurements, visual inspection, performance data and scheduled tests. Condition data can then be gathered at certain intervals, or continuously [1].

\subsection{Levels of Maintenance}

Levels of maintenance of such an engine are 3:

$1^{\text {st }}$ Degree of Maintenance (Organizational Level Maintenance)

Organizational, or O-level maintenance occurs at the organizational unit level, for example by a single maintenance squadron as part of an aircraft wing.

\section{$2^{\text {nd }}$ Degree of Maintenance (Intermediate Level Maintenance)}

Intermediate, or I-level maintenance occurs in specialized backshops that are typically allocated to multiple operating units residing at a common operating location, for example, an entire wing or multiple wings at an operating base.

\section{$3^{\text {rd }}$ Degree of Maintenance (Depot Level Maintenance)}

Depot, or D-level maintenance typically occurs in highly specialized repair depots, or at original equipment manufacturer facilities. These sites are typically not at operating locations, and extensive diagnostic equipment and possibly even manufacturing capabilities exist.

\section{Case Study}

The case study is dealing with a broken fan blade of this engine from the $9^{\text {th }}$ stage of compression. This incident was found incidentally when the engine was sent to the $2^{\text {nd }}$ degree of maintenance in order to fix some erosion issues in another station of the engine. The incident of the broken blade had no detectable effect on engine's performance since its occurrence. Consequently, the incident happened between the two maintenance acts $\left(1^{\text {st }}\right.$ degree of maintenance when the engine was fine and the $2^{\text {nd }}$ degree maintenance when the broken blade was detected).

\section{Data Analysis}

\subsection{Data Selection}

Data for this research were collected from CETADS, a long-term data storage program with the capability of data processing and analysis. These data are regularly recorded from the engine's computer during aircraft's take off [4]. Recordings during take-off are usually selected for such studies, since the engine is operating close to its design point. Moreover, in this case, we are trying to assess the impact of a fractured blade, which will cause minor changes to the sensor measurements. So, in order to be able to detect such a small deviation, we chose to study the engine during the take-off phase, where even a small deviation from the normal values would be more " noticeable " in the sensor recordings, since the engine works over $100 \%$ of its potential, producing the maximum thrust.

Among all CETADS available parameters, the 3 following were chosen to be studied, since they are expected to capture in best the incident under investigation [6]:

- High Pressure Compressor Output Pressure (PB)

- Fan Turbine Inlet Temperature (FTIT)

- Angle of Variable Geometry Guide Blades (RCVVs) 


\subsection{Data Presentation}

All the parameters mentioned above will be dimensionless because these data are confidential for the HAF's aircrafts fleet [5]. In this paper we will only present the methodology we used.

\subsubsection{Burner Pressure (PB)}

More specifically, the diagram (Diagram 1) below shows the change in output pressure (PB) from the high-pressure compressor (HPC) as a function of engine operating hours. The reason we are studying this pressure is that this is expected to be the parameter that is mostly affected by a compressor's error and therefore it is expected to demonstrate noticeable deviations.

But in order to be able to compare and find that the compressor is having an issue, this pressure fluctuation shall be compared against to what it should have had if the compressor had been operating without any problems at all, that is against its healthy condition. Consequently, an engine operation baseline shall be defined which will represent the engine healthy state. It was defined based on the recordings the first year of operation, while the engine was the least degraded with no incidences recorded.

To achieve this, the measured value of pressure was expressed as a function of the engine's rpm. Through this illustration, we were able to find out the relationship between the compressor outlet pressure and rpm and apply it to the appropriate speed.

The next step was to convert this correlation into a correlation of PB with rpm, by the results of this correlations both for the healthy engine state and for the "faulty" engine state are plotted on a common diagram in order to be able to have a direct comparison between each other.

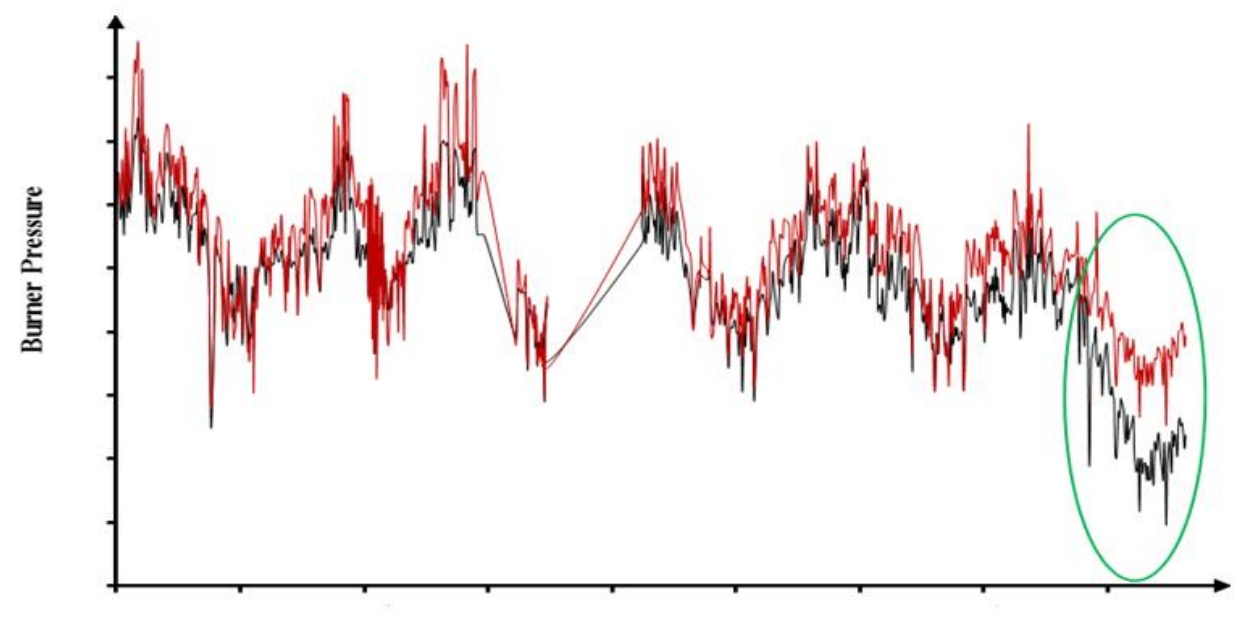

Engine operating time

\section{Diagram 1: $P B=f($ Time). The red $P B$ is the healthy engine \& the black one is the damaged engine}

It is worth noting that in the diagram (Diagram 1) above we find the point where the two graphs start to deviate and mark an operating period for the engine that needs further 
investigation, since it concerns the "suspicious" time period when the incident of blade failure must have occurred.

Zooming in on the point these two graphs are separated enough and marking its last maintenance, the following diagram is drawn (Diagram 2).

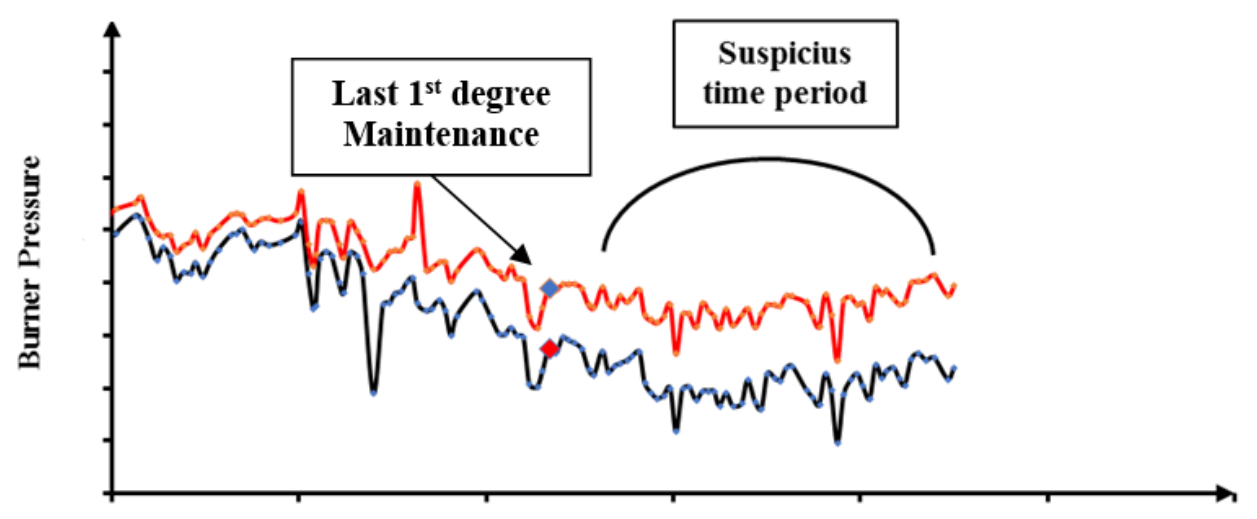

Engine operating time

Diagram 2: $P B=f($ Time). The red $P B$ is the healthy engine \& the black one is the damaged engine, after the last $1^{\text {st }}$ degree of maintenance

In order to have more tangible results the percentage deviation of the measured PB from health $\mathrm{PB}$, as a function of the rpm, is defined based on equation (1) below:

$$
\frac{\Delta(P B)}{P B}=\frac{P B_{M E A S U R E D}-P B_{H E A L T H}}{P B_{H E A L T H}}
$$

The reason we introduced this parameter was to monitor the variation of a single value, against certain limits that can be easily observed. Therefore, by expressing the above formula for the measurements of the current study in percentage format, the following diagram is obtained. 


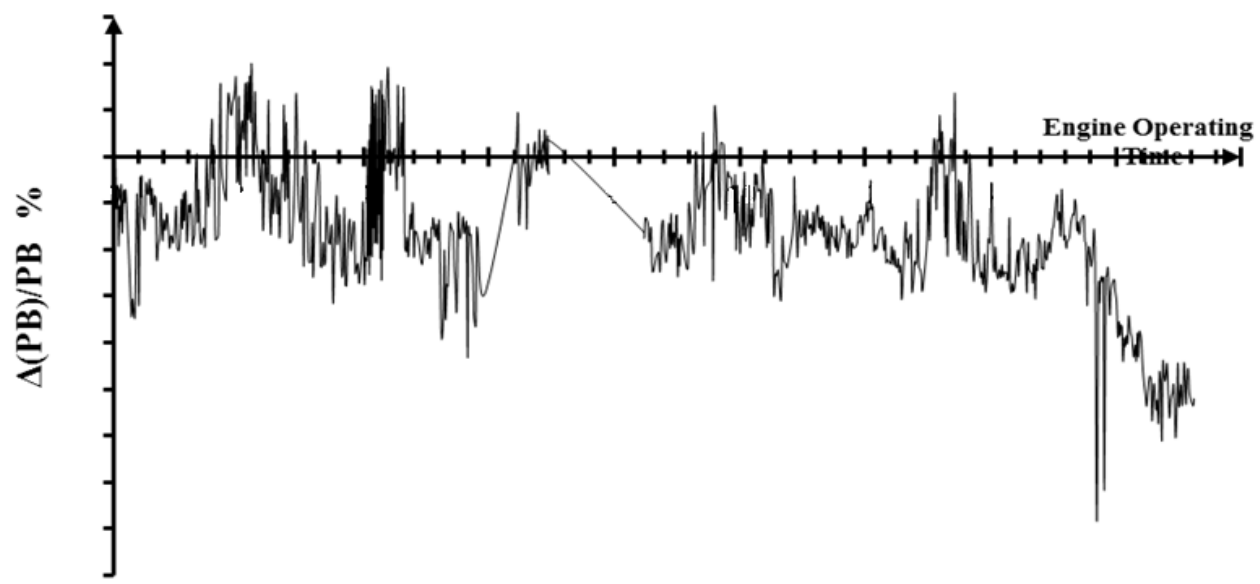

Diagram 3: Percentage change of HPC outlet pressure against engine operating hours.

Diagram 3 shows a clearer picture regarding the outlet pressure deviation between the "healthy" and "faulty" engine operation. Information on diagram 3 indicates clearly the probable time period the blade incident occurred, and in particular on the last period of operation, which confirms initial suspicion.

In the following diagram (Diagram 4) after magnifying this period of time, the following is observed:

- 2 points with 'big' deviation.

- A tendency of increasing the total pressure difference.

- A new engine operating state after deviation is stabilized.

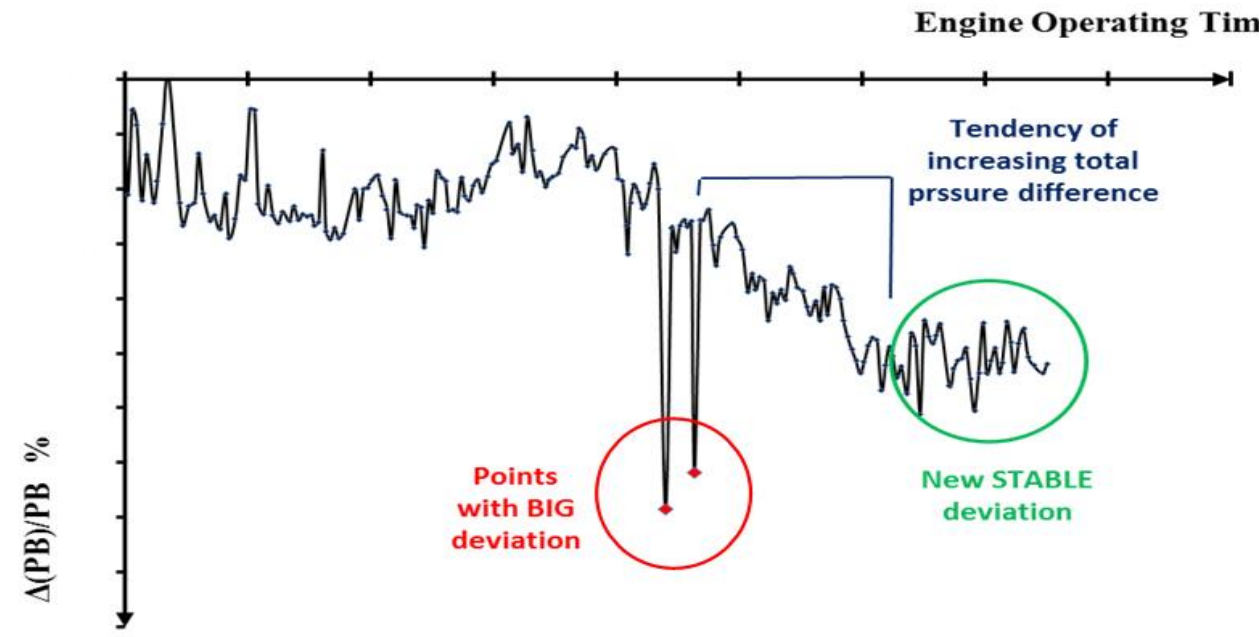

Diagram 4: The percentage deviation of the measured $P B$ from health $P B$, depending on engine operating time, after the last $1^{\text {st }}$ degree maintenance 


\subsubsection{Fan Turbine Inlet Temperature}

The second parameter that was studied, was the temperature measured by the sensors before the hot exhaust stream enters to the low turbine, which is connected with the fan. There are 7 sensors that measure this temperature, a fact that makes this measurement more trusted and reliable. On the following diagram (Diagram 5) the average value of these seven measurements taken by the engine computer, as well as the measurements it should have had if it was operating under normal conditions are demonstrated. The same methodology was followed as in the case of the pressure discussed on the previous subsection.

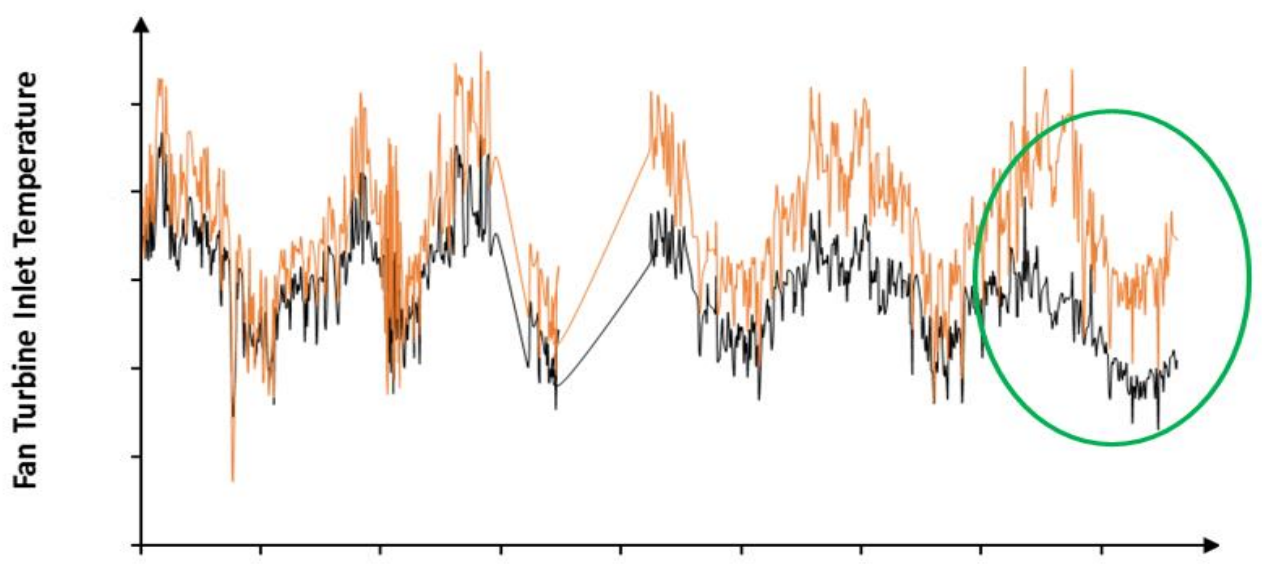

Engine Operating Time

\section{Diagram 5: FTIT=f(Time). The red PB is the damaged engine \& the black one is the health engine.}

Based on Diagram 5, it is clear that during the last hours of the plotted period, there is a greater deviation to measured values than these from a 'healthy' engine operation.

This indication of temperature cannot be considered a fully reliable parameter regarding the detection of the compressor incident under investigation, given that the combustion chamber and the high-pressure turbine placed in-between them are also handling the working medium. However, it is an important indication that something is possibly happening in the engine, which leads to this deviation.

\subsubsection{Engine Reaction}

The operation of the engine is entirely regulated by a Digital Electronic Engine Controller (DEEC), the main function of which is the properly adjustment of the engine in order to deliver what the operator requires. The DEEC unit is programmed to monitor and to stabilize the compression ratio and consequently the compressor output pressure, by changing various engine parameters. One such "handle" parameter for the controller is the variable geometry guides at the high compressor inlet (RCVVs). In order to reject the slightest possibility that the measurements recorded by the engine data logger coming from the sensor measuring the compressor outlet pressure are incorrect, the authors studied the engine controller response to the outlet pressure changes. If there would have been no reaction, that would mean that the problem concerns only the data logger. 


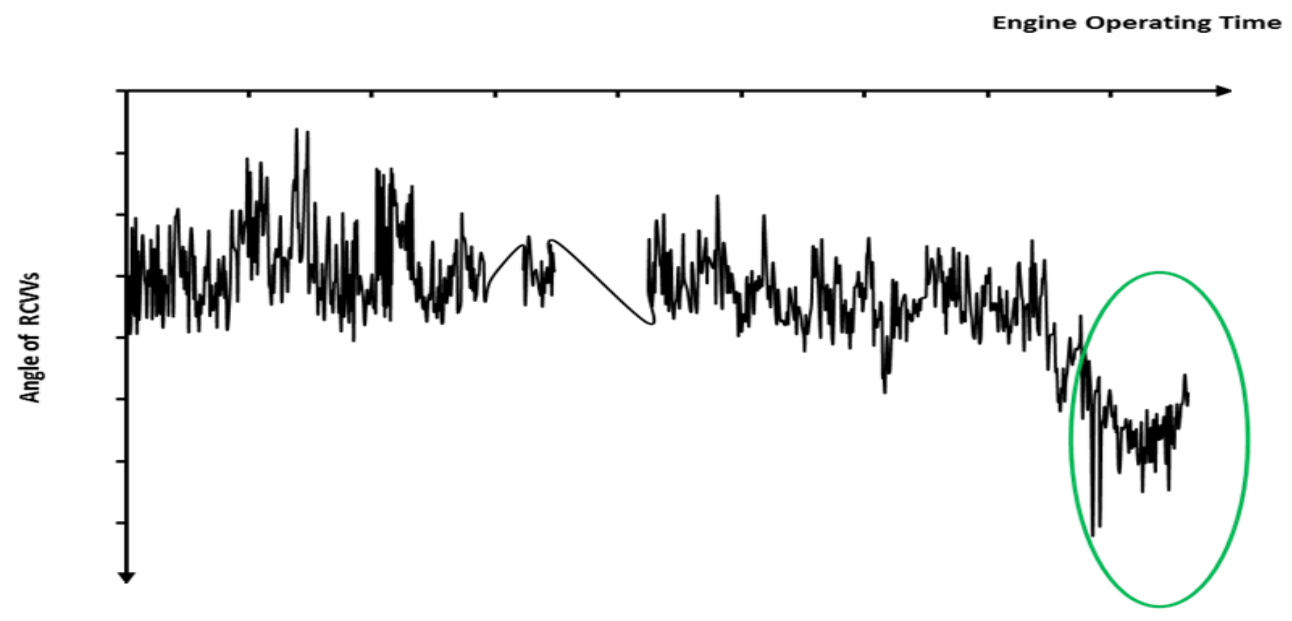

Diagram 6: Angle of RCVVs depending on the engine operating time

On Diagram 6, a similar change, as the one in the pressure, is also recorded for the angle of the RCVVs, which verifies the fact that there is a problem in the compressor and not in the pressure sensor or the data logger. By connecting the compressor outlet pressure reduction, with the RCVVs angle changing, we observe that, when the pressure tends to reduce, the engine changes the RCVVs' angle to reduce the intake air, in order to maintain compression in the desirable levels.

Finally, in the latest RCVVs angle measurements, after the new value stabilization, there is a tendency to return to its " normal " values.

\section{Conclusions}

It is always a challenge for a big organization such as the HAF (Hellenic Air Force) to engage various units in synergetic schemes in order to multiply its capabilities. In our case we had a bottom up approach where the need and the way to be covered was located at a lower management level, it was communicated appropriately upwards, approvals were granted and the goal was achieved.

The purpose of this paper was to study the tendency of the thermodynamic parameters of the working medium and the correlation of them with engine failure. More specific, after studying the incident of the broken blade that occurred on a fighter jet engine, we came to the following conclusions:

$>$ We found the possible time-period the incident happened.

$>$ We concluded that the engine after the incident had balanced at a new output pressure with a loss of pressure about $1-1.5 \%$.

$>$ The results of the investigation were used by the Incident Investigation Committee during the effort to identify the time the incident happened.

$>$ It was revealed that there were no gaps or omissions in the technical support procedures that required corrective action - sanctions

$>$ Conditions for conducting corresponding studies on any relevant future failures were created. 


\section{References}

[1] https://www.fiixsoftware.com/condition-based-maintenance/ (2019)

[2] N. Aretakis, I. Roumeliotis, A. Alexiou, C. Romesis, K. Mathioudakis, Journal of Engineering for Gas Turbines and Power APRIL 2015, Vol. 137 I 041203-1 (2015)

[3] F100-PW-229 Proposal for the New Fighter Aircraft for the Hellenic Air Force (1998)

[4] Engine Monitoring System Reference Guide, F100-PW-229 (2003)

[5] A.J.Volponi, Gas Turbine Parameter Corrections (1999)

[6] Performance-analysis-based gas turbine diagnostics, Department of Power, Propulsion and Aerospace Engineering, School of Engineering, Cranfield University, Bedfordshire, UK (2002)

[7] Anthony Giampaolo, Gas Turbine Engineering Handbook, Principles and Practices, Third Edition, (2006) 